\title{
Breakdown of the Kondo Effect in Critical Antiferromagnets
}

\author{
Davide Controzzi \\ Department of Physics, Theoretical Physics, University of Oxford, \\ 1 Keble Road, Oxford OX1 3NP, UK
}

\begin{abstract}
The breakdown of the Kondo effect may be the origin of the anomalous properties of the heavyfermion compounds at low temperatures. We study the dynamics of one impurity embedded in an antiferromagnetic host at the quantum critical point and show that the impurity is not screened and develops a power law correlation function. This suggests that the breakdown of the Kondo effect can simply be a consequence of the system's proximity to the quantum critical point.

PACS numbers: 71.10.Hf, 71.27.+a,75.20.Hr
\end{abstract}

A number of heavy-fermion systems present very peculiar non-Fermi-liquid properties which have been suggested to be a consequence of the proximity of the antiferromagnetic quantum critical point (QCP)1. The weak-coupling approach to quantum phase transitions 2 , which describes many ferromagnetic compounds fairly well fails in describing the temperature exponents of various thermodynamic and transport properties of these materials 5 . Recent neutron scattering experiments in $\mathrm{CeCu}_{6-x} \mathrm{Au}_{x}$ havesuggested that the failure of this approach can be related to the unusual energy dependence of the spin susceptibility 3 . The measured $\omega / T$ scaling of the spin susceptibility:

$$
\chi(\omega, T)=\frac{1}{T^{\alpha}} f\left(\frac{\omega}{T}\right)
$$

was fitted with:

$$
\chi^{-1}=c^{-1}\left[f(\mathbf{q})+(-i \omega+a T)^{\alpha}\right]
$$

where $\alpha \simeq 0.8$, while in the weak coupling approach $\alpha=1$. This result suggests that the anomalous non-Fermi-liquid behavior in these compounds may have a local origin. One possible interpretation is that the screening that leads to the Kondo effect is incomplete as a consequence of the proximity to the QCP3.

A first analysis of the Kondo effect in systems close to a QCP was performed more than twenty years ago by Larkin and Mel'nikovl. They showed that the Kondo screening is not effective in itinerant electron ferromagnets close to the QCP. At the present there is no theory of the Kondo effect at the antiferromagnetic QCP. Some authors have suggested that disorder is an essential ingredient for the breakdown of the Kondo effect, and that it is therefore the origin of the anomalous properties of heavy-fermion materials. In the Kondo-disorder model suggested by Miranda, Dobrosavljević, and Kotliard, a randomness in the distribution of the hybridization matrices leads to a broad distribution of the Kondo temperatures with a tail that goes to zero. Some spins then remain unscreened at all temperatures and they can be the origin of anomalies in the thermodynamics and transport properties. A different approach is based on the metallic spin glass model introduced by Sengupta and Georges and Sachdev, Read and Oppermann 9 . In these studies it was shown that, at the critical point, the spin fluctuation spectrum is non-Fermi-liquid-like, and the impurity spins develop a power law correlation function in time. Nevertheless the rôle of disorder in heavy-fermion materials and, in general, of the non-Fermi liquid properties of metals is still a hotly debated question. It is therefore important to understand whether these results are specific to the QCP under consideration, or if they survive also in clean systems.

Anotherpossible origin of the anomalous local susceptibility is the formation of a pseudo-gap in the single particle spectrum 12. In quantum critical systems that show superconductivity the pseudo-gap can be generated by superconducting fluctuations but, in general, the origin of the pseudo-gap in critical itinerant antiferromagnets is not flear at the moment and has been suggested mainly on the basis of studies employing Dynamical Mean Field Theory 13 .

One way to represent a system when the Kondo effect breaks down js ta consider a spin living in a cavity in the presence of an effective Gaussian Weiss field $h$ with correlation function 10 . 11 :

$$
\left\langle T h_{a}(\tau) h_{b}(0)\right\rangle \sim \frac{\delta_{a, b}}{\tau^{2-\epsilon}}
$$

Integrating over the Gaussian field $h$ we get the following contribution to the effective action of the local spin:

$$
I=g \int d \tau \frac{S^{a}(\tau) S^{a}\left(\tau^{\prime}\right)}{\left|\tau-\tau^{\prime}\right|^{(2-\epsilon)}}
$$

where $g$ is the coupling constant. In itinerant antiferromagnets like $\mathrm{CeCu}_{6-x} \mathrm{Au} \mathrm{u}_{x}$ this action has to be combined with the usual Kondo screening. Such a model was analyzed by Sengupta10, who used an $\epsilon$-expansion and found that the model with Kondo screening possesses an unstable non-Fermi-liquid fixed point. In the leading order in $\epsilon$ the exponent 
of the spin-spin correlation function at the fixed point coincides with the exponent for the model without Kondo screening. For the latter case the results of Sengupta coincide with a naïve scaling analysis which gives $[S]=\tau^{-\epsilon / 2}$ and $\langle S(\tau) S(0)\rangle \sim \tau^{-\epsilon}$ for the spin-spin correlation function. In frequency space we have $\chi_{0}^{-1}(\omega) \sim(-i \omega)^{1-\epsilon}$. This approach naturally leads to $\omega / T$ scaling of the spin susceptibility of the form (11) with

$$
\alpha=1-\epsilon
$$

In this paper we discuss the behavior of a single impurity in an antiferromagnetic host close to the QCP. Our aim is to obtain Sengupta's phenomenological theory from a microscopic approach to a critical antiferromagnet. This would show that the Kondo effect in the system is not complete. We first analyze a model of itinerant electron antiferromagnets (IEAF), which is a possible model for heavy-fermion systems, and then briefly consider also a model for an insulator (IAF). The results are very similar to those obtained for metallic spin glasses and suggest that proximity to the QCP is enough to suppress Kondo screening. We use a path integral approach and represent the bulk as a bosonic field $\mathbf{n}(\mathbf{x}, \tau)$ interacting with the impurity via the following action:

$$
S=S^{b u l k}+J \int d \tau \mathbf{n}(0, \tau) \cdot \mathbf{S}(\tau)
$$

where

$$
\begin{array}{r}
S^{b u l k}=\int d \omega d^{D} k\left[\frac{1}{2} \mathbf{n}(\mathbf{k}, \omega) D_{0}^{-1}(\mathbf{k}, \omega) \mathbf{n}(-\mathbf{k},-\omega)\right]+ \\
g \int d \tau d^{D} x\left(\mathbf{n}(\mathbf{x}, \tau)^{2}\right)^{2}
\end{array}
$$

The form of the bare propagator, $D_{0}(\mathbf{k}, \omega)$, depends on the host material.

In the case of the $D$-dimensional IEAF the spin-spin correlation function of the host can be obtained in the random phase approximation and is 2 :

$$
D_{0}(\mathbf{k}, \omega)^{-1}=|\omega|+k^{2}+\delta
$$

where $\delta$ vanishes at the critical point. In relation to the bulk properties the $\mathbf{n}$-field can be treated as Gaussian if the effective dimensionality of the bulk theory exceeds the upper critical dimension. This condition is satisfied for the IEAF QCP, for $D>2$ (which is the case that we shall discuss in this paper), while for the IAF the quartic term renormalizes the bulk propagator giving rise to an anomalous power.

Treating the bulk field as Gaussian and integrating it out we obtain an effective theory of the form (4), with:

$$
\epsilon=2-D / 2, \quad \alpha=D / 2-1
$$

In order to check whether the interaction between the modes of the $\mathbf{n}$-field, irrelevant for the bulk properties, remains irrelevant for the impurity, we have calculated the quartic term in the impurity effective action:

$$
\begin{array}{r}
S_{\text {eff }}[\mathbf{S}]=\frac{1}{2 J} \int d \tau \mathbf{S}(\tau) D\left(0, \tau-\tau^{\prime}\right) \mathbf{S}\left(\tau^{\prime}\right)+ \\
g \int d \tau_{1} d \tau_{2} d \tau_{3} d \tau_{4} \mathbf{S}\left(\tau_{1}\right) \mathbf{S}\left(\tau_{2}\right) \mathbf{S}\left(\tau_{3}\right) \mathbf{S}\left(\tau_{4}\right) \Gamma\left(\tau_{1}, \tau_{2}, \tau_{3}, \tau_{4}\right)
\end{array}
$$

where

$$
\begin{aligned}
\Gamma\left(\tau_{1}, \tau_{2}, \tau_{3}, \tau_{4}\right)=\int d \tau d \tau^{\prime} d^{D} x d^{D} x^{\prime} D\left(\mathbf{x}, \tau_{1}-\tau\right) \\
\qquad D\left(\mathbf{x}, \tau_{2}-\tau\right) g\left(\mathbf{x}-\mathbf{x}^{\prime}, \tau-\tau^{\prime}\right) D\left(\mathbf{x}^{\prime}, \tau-\tau_{3}\right) D\left(\mathbf{x}^{\prime}, \tau-\tau_{4}\right)
\end{aligned}
$$

$g(\mathbf{x}, \tau)$ is the renormalized bulk vertex and $D(\mathbf{x}, \tau)$ the renormalized bulk propagator. As mentioned above, for this model the propagator is not renormalized and the same is true for the interaction, so $\Gamma$ scales like $\Gamma \sim \tau^{1-3 / 2 D}$ and the spin operator like $S \sim \tau^{D / 4-1}$. Then the four-spin interaction scales like $\tau^{1-D / 2}$, and is irrelevant for $D>2$, which is the case of interest.

For $\mathrm{CeCu}_{6-x} \mathrm{Au}_{x}$ the spin fuctuation spectrum is anisotropic in space 14 and the coefficient of the quadratic term is very small in one direction 3 . In the framework of this model such anisotropy can only be obtained introducing it at a bare level. We therefore consider also: 


$$
D_{0}^{-1}(\mathbf{k}, \omega)=|\omega|+A k_{\perp}^{2}+B k_{\|}^{4}+\delta
$$

In this case we obtain the same results with an effective dimension given by:

$$
D=D_{\text {eff }}=5 / 2 \text {. }
$$

This is related to the fact that, in the action, the soft direction provides less phase space and counts as 'half a dimension'. We then find from (9) that $\alpha=0.5$ in the isotropic (3D) case, and $\alpha=0.25$ in the anisotropic one. This simple analysis shows that in critical itinerant electron antiferromagnets, described by the model (7) with the bare propagator (8), the screening effect is incomplete and the impurity develops a power law correlation function. The effect however is too strong to describe the observed behavior of $\mathrm{CeCu}_{6-x} \mathrm{Au}_{x}$.

It is interesting to compare these results with the behavior of an insulator. The QCP in such a system is described by the $(\mathrm{D}+1)$-dimensional $\mathrm{O}(3)$ non-linear sigma model with a special value of the coupling constant. At the QCP the propagator has the form:

$$
D(\mathbf{k}, \omega)^{-1}=\left(\omega^{2}+k^{2}\right)^{1-\eta / 2}
$$

Thus in D dimensions we have:

$$
\alpha=D-2+\eta
$$

In $D=3, \eta=0$ which gives $\alpha=1$ (modulo logarithmic corrections). For $D=2$ the index $\eta$ can be obtained by taking into account that the (2+1)-dimensional QCP is in the same universality class as the ferromagnetic phase transition point in a three-dimensional Heisenberg ferromagnet. For the latter system the exponent $\eta$ is known to be 0.03115 . Thus in $\mathrm{D}=2$ we have:

$$
\alpha=\eta
$$

It is reasonable to expect that for $D=2.5$ the index $\eta$ is also small, so we get the estimate $\alpha \approx 0.5$.

In summary, we have studied the the behavior of one impurity in two magnetic systems close to the antiferromagnetic quantum critical point. As a consequence of the proximity of the QCP, the Kondo effect doesn't occur, and the impurity develops a power law correlation function in time. The emerging picture is very similar to the one proposed to describe the non-Fermi-liquid behavior in heavy-fermion materials and to that found in some works on metallic spin glasses. It suggests that a system, approaching the QCP, undergoes a phase transition from a Kondo phase, with Fermi liquid properties, to a non-Fermi-liquid phase, dominated by field fluctuations. This suggests that incomplete Kondo screening and power law dependence of the spin-spin correlation function are not necessarily related to disorder, but can be simply consequences of proximity to the AFM critical point.

It is a pleasure to thank Alexei M. Tsvelik for many interesting discussions on the subject and for comments on the draft, and C.Hooley for reading the manuscript.

${ }^{1}$ B.Andraka and A.M.Tsvelik, Phys. Rev. Lett. 67, 2886 (1991).

2 J.A.Hertz,Phys. Rev. B 14, 1165 (1976); A.J.Millis, Phys. Rev. B 48, 7183 (1993).

${ }^{3}$ Schröder et alt., Phys. Rev. Lett. 80, 5623.

${ }^{4}$ S.R.Julian et alt.,J.Phys:Cond.Matt.,8, 9675; C.Pfleiderer et alt., Phys. Rev. B 558330.

${ }^{5}$ See for instance H.von Löhneysen, J.Phys:Cond.Matt.,8,9689.

${ }^{6}$ A.I.Larkin and V.I.Melnikov, JEPT, 34, 656 (1972).

${ }^{7}$ E.Miranda, V.Dobrosavljević, G.Kotliar Phys. Rev. Lett. 78, 290 (1997).

8 A.M.Sengupta and A.Georges, Phys. Rev. B 52, 10295 (1995).

${ }^{9}$ S.Sachdev, N.Read and R.Oppermann Phys. Rev. B 52, 10286 (1995).

${ }^{10}$ A.Sengupta, cond-mat 9707316.

${ }^{11}$ P.Coleman, Proceeding to SCES98, Physica B 353, 258 (1999).

${ }^{12}$ K.Ingersent and Q.Si, cond-mat/9810226; Q.Si, J.L. Smith and K.Ingersent, Int.J.Mod.Phys.B 13, 2331 (1999).

${ }^{13}$ A.Georges, G.Kotliar, W.Krauth and J.Rozenberg, Rev. Mod.Phys 68, 13 (1996).

${ }^{14}$ A.Rosch, A.Shröder, O.Stockert and H.von Löhneysen, Phys. Rev. Lett. 79, 159 (1997).

${ }^{15}$ C.Itzykson and J-M.Drouffe, Statistical field theory, Cambridge University Press (1989). 